Expl oring deut eri um beam oper at $i$ on and the behavi or of the co- extracted el ectron cur rent i $n$ a negat $i$ ve- $i$ on- based neut $r$ al beam i nj ect or

\begin{tabular}{|l|l|}
\hline $\begin{array}{l}\text { j our nal or } \\
\text { publ i cat i on } \mathrm{t} \text { i t l e }\end{array}$ & Nucl ear Fusi on \\
\hline vol une & 59 \\
\hline number & 7 \\
\hline page $r$ ange & 076009 \\
\hline year & $2019-05-24$ \\
\hline URL & ht t p: //hdl . handl e. net /10655/00012575 \\
\hline
\end{tabular}




\title{
Exploring Deuterium Beam Operation and the Behavior of the Co-Extracted Electron Current in Negative-Ion-Based Neutral Beam Injector
}

\author{
K. Ikeda ${ }^{1}$, K. Tsumori ${ }^{1,2}$, H. Nakano ${ }^{1,2}$, M. Kisaki ${ }^{1,2}$, \\ K. Nagaoka ${ }^{1,3}$, S. Kamio ${ }^{1}$, Y. Fujiwara ${ }^{1}$, Y. Haba ${ }^{3}$, \\ and M. Osakabe ${ }^{1,2}$ \\ ${ }^{1}$ National Institute for Fusion Science (NIFS), Toki, 509-5292, Japan \\ 2 SOKENDAI (Graduate School for Advanced Studies), Toki 509-5292, Japan \\ ${ }^{3}$ Graduate School of Engineering, Nagoya University, Nagoya 464-8603, Japan \\ E-mail: ikeda.katsunori@lhd.nifs.ac.jp
}

20 Feb. 2019

\begin{abstract}
Achievements of the deuterium beam operation of the negative-ion-based neutral beam injector (N-NBI) in the Large Helical Device (LHD) are reported. In the beam operation in LHD-NBIs, both hydrogen $(\mathrm{H})$ and deuterium (D) neutral beams were generated by changing the operation gas using the same accelerator. The maximum accelerated deuterium negative ion current $\left(I_{a c c(D)}\right)$ reaches $46.2 \mathrm{~A}$ from two beam sources with the averaged current density being $190 \mathrm{~A} / \mathrm{m}^{2}$ for $2 \mathrm{sec}$, and the extracted electron to accelerated ion current ratio $\left(I_{e} / I_{a c c(D)}\right)$ increases to 0.39 using 5.6 V high bias voltage in the first deuterium operation in 2017. Increase of electron density in the vicinity of the plasma grid surface, which is considered the main reason of increasing co-extracted electrons in a beam, is confirmed by half-sized research negative ion source in the neutral beam test stand in NIFS. The deuterium negative ion density is also larger than the hydrogen negative ion density in the vicinity of plasma grid surface using the same discharge condition. In the latest experimental campaign in 2018, the $I_{a c c(D)}$ increases to $55.4 \mathrm{~A}$ with the averaged current density being $233 \mathrm{~A} / \mathrm{m}^{2}$ for $1.5 \mathrm{sec}$ using the shot extraction gap length. The low $I_{e} / I_{a c c(D)}$ of 0.31 can be maintained by high discharge power. The various parameters mentioned above are defined in detail below.
\end{abstract}

Keywords: negative-ion, co-extracted electron, NBI

Submitted to: Nucl. Fusion 


\section{Introduction}

Production of high intensity negative ion beams with low co-extracted electrons is a common issue for a negative-ion-based neutral beam injector (N-NBI) for heating and current drive in magnetically confined fusion device. Deuterium N-NBI with the beam energy of $500 \mathrm{keV}$ in JT-60U [1,2] and hydrogen N-NBI with the beam energy of $180 \mathrm{keV}$ in LHD [3-7] worked in Japan from 1996 and from 1998, respectively, with the latter reaching the full power specification for $2 \mathrm{sec}$ beam in 2003 [8]. An N-NBI generating $1 \mathrm{MeV}$ deuterium ions for 1 hour being developed for ITER [9,10], and the experiment of SPIDER [11,12] has started in 2018 in the ITER-NBI test facility in Padova. In the ITER-NBI, a deuterium beam of $40 \mathrm{~A}$ is planned with the beam energy of $1 \mathrm{MeV}$ in the D-D and the D-T experiment phases, but the NBI will be operated at $870 \mathrm{keV}$ and 46 A hydrogen beam in early hydrogen experiment phase [10]. Increase of co-extracted electrons for deuterium negative ion beam operation has been observed by the ITER half size source [13], which is an important issue for the realization of ITER-NBI. Therefore, optimization of generation negative ions and suppression of co-extracted electron current for both hydrogen and deuterium are required.

In the LHD, the first deuterium beam operation of the N-NBI was initiated in 2017 [14]. We generated both hydrogen and deuterium negative-ion beams in N-NBIs by changing the operation gas using the same accelerator. After the LHD experiment campaign, we investigated the characteristics of the negative ion density and the electron density by a research negative ion source (RNIS) in the neutral beam test stand (NBTS) in NIFS. Comparison of the beam properties such as the extracted negative ion current and the co-extracted electron current and source properties with $\mathrm{H}_{2}$ and $\mathrm{D}_{2}$ gases will clarify the production and extraction mechanism of the negative ions. This should contribute to the ITER and future NBIs.

This paper describes the results of hydrogen and deuterium beam operations in LHD-NBI. Changes in the negative ion current and electron current clearly appeared when the discharge gas was changed from hydrogen to deuterium. We will show the electron current and electron-ion current ratio, which clearly depends on the arc discharge power. We also show changes in the negative ion density and the electron density in the vicinity of cesiated metal surface in the negative ion source, and will discuss the similarity to the deuterium negative ion current and the electron current, respectively.

Various parameters mentioned in the abstract and in the following text are defined below. It is to be noted that the typical time of beam extraction was between $1.5 \mathrm{sec}$ and $2 \mathrm{sec}$ in high power beam operation in LHD-NBI. Only small variations $(<5 \%)$ of the various parameters discussed below occurred over that period and the values quoted are averaged over the beam extraction period.

- Deuterium negative ion current $\left(I_{a c c(D)}\right)$ :This is the electrical current to the grounded grid of the accelerator and the components downstream of the grounded grid when the ion source is operated with $\mathrm{D}_{2}$ gas. That current will contain some 
electrons created by stripping in the acceleration gap and some electrons escaping through the extraction grid into the acceleration gap. The fraction of electrons is estimated to be typically $\approx 13 \%$.

- Hydrogen negative ion current $\left(I_{a c c(H)}\right)$ :This is the electrical current to the grounded grid of the accelerator and the components downstream of the grounded grid when the ion source is operated with $\mathrm{H}_{2}$ gas. That current will contain some electrons created by stripping in the acceleration gap and some electrons escaping through the extraction grid into the acceleration gap. The fraction of electrons is estimated to be typically $\approx 10 \%$.

- Electron current $\left(I_{e}\right)$ :This is the current to the extraction grid, which is defined $I_{e}=I_{e x}-I_{a c c}$, here $I_{e x}$ is the electrical current to the extraction grid. Electrons extracted from the ion source and electrons created by the stripping of extracted negative ions in the extraction gap and the aperture in the extraction grid are deflected onto the extraction grid by the magnetic field of the electron deflection magnets embedded in the grid. It was estimated that $\approx 97 \%$ of those electrons are absorbed by the extraction grid, and that $3 \%$ escape into the acceleration gap [15]. The number of electrons created by stripping is typically $\approx 3 \%$, so the current to the extraction grid is $\approx 106 \%$ of the extracted electron current.

- Averaged negative ion current density:This is the deuterium or hydrogen negative ion current (defined as above) divided by the area of the apertures in the plasma grid.

- Filling pressure:The gas flow into the ion source is adjusted to give a chosen "filling pressure", which is the pressure in the ion source when the discharge is not operating (and there is no ion extraction). The gas flow is kept constant before and during source operation and ion extraction, but the gas pressure in the source will change significantly during source operation because the gas is heated by the discharge, there will be significant dissociation of the gas, and because ions are extracted from the source.

\section{Ion Source Setting and Operation Conditions}

\subsection{Deuterium operation in LHD-NBI}

The first deuterium experiment of the LHD was initiated after reference plasma discharge using hydrogen. Two positive-ion-based NBIs (P-NBI) and three N-NBIs are equipped in the LHD [16]. Before the deuterium experiment, the beam energy of P-NBI was upgraded from $40 \mathrm{keV}$ to $60 \mathrm{keV}$ and to $80 \mathrm{keV}$ in the beam line four (BL4) and the beam line five (BL5), respectively, to concentrate the deuterium beam operation. The beamlet focusing condition is also optimized for deuterium by wide gap distance [17]. The nominal injection power increased from $6 \mathrm{MW}$ to $9 \mathrm{MW}$ in each beam line, which are contributed to increase ion temperature in deuterium discharges. 
On the other hand, the beam energies in three N-NBIs are not increased to suppress neutron generation by $\mathrm{D}-\mathrm{D}$ reaction of beam component within permissible neutron budget $\left(2.1 \times 10^{19} /\right.$ year $\left.[18,19]\right)$ in LHD. Two hydrogen negative ion sources are equipped in one beam line [20]. The nominal beam energy is $180 \mathrm{keV}$ in three beam lines, and an acceleration voltage is applied between an extraction grid (EG) and a grounded grid (GG), which is $70 \mathrm{~mm}$ for the first beam line (BL1) and $60 \mathrm{~mm}$ for the second beam line (BL2) and the third beam line (BL3) optimized for hydrogen operation. For this reason, the maximum injection power can be obtained by hydrogen beam operation. Therefore, the highest ion temperature was achieved using a hydrogen beam from N-NBIs and a deuterium beam from P-NBI in LHD deuterium experiment campaign. We uses a slot type GG $[6,21]$ in BL1 and BL3. The GG is equipped with the 55 slots, and is divided into five segments. The slot width is $14 \mathrm{~mm}$ with the transparency of $76 \%$ in BL3, which is much higher than the transparency of $50 \%$ for a conventional aperture type of GG (diameter of the aperture $=16 \mathrm{~mm}$ and 770 apertures in one source) in BL2. Improvement in transparency reduces the thermal load on the GG due to the peripheral components of negative ion beam, and reduces the number of electrical breakdowns in the accelerator. However, since the focusing conditions of the slit type GG are different in the vertical direction and the horizontal direction, the aperture shape of the steering grid on the back surface of the EG is arranged to the racetrack shape [22] to adjust beam divergence. The extraction gap length between a plasma grid (PG) and an the EG is also an important setting in a perveance condition of an ion beam. In the BL3, the extraction gap length of of $8 \mathrm{~mm}$ which is a setting for extracting the maximum current of hydrogen negative ions on the design was used in first deuterium operation in 2017 (Table 1). Typical extraction voltage between the PG and EG is $10 \mathrm{kV}$ for the $170 \mathrm{kV}$ acceleration voltage in hydrogen operation. Table 1 also shows summary of the $I_{a c c(H)}$, the $I_{a c c(D)}$, the $I_{e} / I_{a c c(H)}$ and the $I_{e} / I_{a c c(D)}$. The $I_{a c c(D)}$ is lower than the The $I_{a c c(H)}$ using the same accelerator in all beam lines in 2017. The current reduction rates are from 0.5 to 0.75 . The ratios of $I_{e} / I_{a c c(D)}$ increases in the deuterium operation, which are from 0.39 to 0.54 .

A negative ion current and electron current are obtained by electrical current measurement in all NBI injectors, and also the optical emission intensity from cesium (Cs) neutral is observed by a wide range spectrometer. However, details of the plasma parameters in the vicinity of the $\mathrm{PG}$ surface in the injector source are not observed in LHD-NBI. Therefore, the difference of source parameters between hydrogen and deuterium is studied using the RNIS in the NBTS. In 2018 operation campaign, the extraction gap length is set $7 \mathrm{~mm}$ in BL3 in order increase the negative ion current after investigation of negative ion density and electron density in the RNIS in the test stand in NIFS. The $I_{a c c(D)}$ increases to $55.4 \mathrm{~A}$, and the $I_{e} / I_{a c c(D)}$ could be suppressed to 0.31 . 


\subsection{RNIS and diagnostic systems in NBTS}

The RNIS is also equipped with a three-stage grid system with an arc discharge type negative ion source. The gird consists of two segments, and the GG uses slot type similar to the LHD-NBI. The beam width is the same as that of the LHD-NBI beam. Since the number of GG slots is 28 in RNIS, the scale factor compared with LHD-NBI is approximately 0.5. Although a hydrogen beam of $120 \mathrm{keV}$ can be generated at the NBTS in NIFS, neutron generation using $\mathrm{D}^{-}$beam acceleration is not permitted in the test stand, but the characteristics of arc discharge plasma using hydrogen and deuterium may be measured.

Various measurement devices are installed in the vicinity of the PG surface with the distance of $19 \mathrm{~mm}$ in the RNIS [23]. A negative ion density in both hydrogen and deuterium and an electron density is obtained by a Cavity-ring-down (CRD) method [24] and a millimeter wave interferometer (mmIF) [25], respectively. Since both density diagnostics are installed parallel to the PG surface in the minor axis direction of the arc chamber, measured densities are line averaged values. A plasma potential is obtained by a Langmuir probe (LP) $[26,27]$. Behavior of Cs neutral particles near the PG surface can be obtained by an optical emission spectroscopy (OES) [28,29] and a Cs laser absorption spectroscopy [23].

\section{Change of Negative Ion Density and Electron Density by Gas Change Experiment}

Before the deuterium operation, the negative ion source was operated by hydrogen for 3 months in LHD. The $I_{a c c(H)}$ increase over $70 \mathrm{~A}$ in all injectors (see Table 1), and the total port through power of $15 \mathrm{MW}$ was injected by 3 beam lines. The ratio of $I_{e} / I_{a c c(H)}$ is 0.3 or less, and it is extremely favorable as 0.23 in BL3. At this time, the bias voltage was $3 \mathrm{~V}$ and the filling pressure was $0.3 \mathrm{~Pa}$. In the hydrogen operation, since Cs is sufficiently coated on the surface of the PG, the Cs emission intensity did not change in 2-second beam operation even when Cs supply was stopped.

Figure 1 shows the shot history of the negative ion current (squares), the electronion current ratio (circles), and the optical emission signal from Cs neutral (diamonds) during the operation gas changing from $\mathrm{H}_{2}$ to $\mathrm{D}_{2}$ in the negative ion source in LHD-NBI. Deuterium gas is filled after evacuating the buffer tank and the feeder pipe of the gas

supply unit. Replacement work has been done on site since the radiation level of LHD equipment has been reduced over the weekend. In order to adjust the arc discharge in the first deuterium operation, the supply of Cs was stopped after gas replacement. The source filling pressure was $0.3 \mathrm{~Pa}$ and the bias voltage is set to $3 \mathrm{~V}$ which is the same value in hydrogen operation.

Deuterium beam extraction is initiated using low arc discharge power $\left(P_{\text {arc }} \sim 50\right.$ $\mathrm{kW} /$ source). The arc discharge power and the beam energy increase to $120 \mathrm{~kW} /$ source and $150 \mathrm{keV}$, respectively, until the shot number of 304242. The $I_{a c c(D)}$ increases from 8 
A to 35 A during this initial conditioning. Cs layer covering the PG surface is important for the production of negative ions. A Cs optical intensity $(852.1 \mathrm{~nm})$ normalized by the hydrogen/deuterium Balmer- $\gamma$ intensity measured by OES in arc discharge phase, which is an index of the Cs neutral density near the PG surface [29], clearly increases after initiation of $\mathrm{D}^{-}$operation. The temperature of the $\mathrm{PG}$ is $230{ }^{\circ} \mathrm{C}$ in both $\mathrm{H}$ and $\mathrm{D}$ discharges. The higher Cs density in deuterium discharges compared to that in hydrogen discharges may be due to higher Cs sputtering by the heavier deuterium from the ion source walls and the plasma grid. The $I_{e} / I_{a c c(D)}$ increases from 0.7 to 1 and then it immediately decreases to 0.5 by additional Cs feeding, which recovers the surface Cslayer. Cs seeding is effective to increase the $I_{a c c(D)}$ to $40 \mathrm{~A}$. However, $\mathrm{D}^{-}$current did not recover to $\mathrm{H}^{-}$current level $\left(I_{a c c(H)}=70 \mathrm{~A}\right)$.

In order to investigate the reasons for the reduction of negative ion current and the increase of electron current in deuterium, the negative ion density and the electron density vicinity of the PG surface are investigated by the RNIS in the NBTS. Figure 2 shows the shot history of the negative ion density $\left(n_{H^{-}}\right.$for hydrogen and $n_{D^{-}}$for deuterium: squares), electron density ( $n_{e}$ : circles), and the optical signal of Cs neutral normalized by Balmer- $\gamma$ emission (diamonds) during the changing of the operation gas from $\mathrm{H}_{2}$ to $\mathrm{D}_{2}$. The gas replacement procedure is the same as LHD-NBI. Since the neutron production does not occur in the NBTS without beam extraction, deuterium discharge initiated immediately after gas replacement. In this situation, we can continuously supply Cs into the arc chamber with an oven temperature of $180{ }^{\circ} \mathrm{C}$. The filling pressure and the bias voltage were set at $0.3 \mathrm{~Pa}$ and at $2.5 \mathrm{~V}$, respectively, the same as hydrogen operation. The arc power is controlled to $50 \mathrm{~kW}$, but it is temporarily reduced to $30 \mathrm{~kW}$ immediately after gas change, and then it recovers to $50 \mathrm{~kW}$ in a few shots. In the hydrogen phase, $n_{H^{-}}=3.5 \times 10^{17} \mathrm{~m}^{-3}$ is stably generated and $n_{e}$ is also stable at $2 \times 10^{17} \mathrm{~m}^{-3}$, which is lower than $n_{H^{-}}$. However in a deuterium discharge, $n_{D^{-}}$is $4.6 \times 10^{17} \mathrm{~m}^{-3}$, which is $30 \%$ higher than the negative ion density in hydrogen, and $n_{e}$ is $6.5 \times 10^{17} \mathrm{~m}^{-3}$, which is larger than $n_{D^{-}}$, and $>2.2$ times higher than in the hydrogen discharge. Thus it seems that the increase of $n_{e} / n_{D^{-}}$is mainly responsible for the increase in $I_{e} / I_{a c c(D)}$. The need to maintain charge neutrality in the plasma in the vicinity of the $\mathrm{PG}$ means that the positive ion density must be increased in deuterium discharges compared to that in similar hydrogen discharges, i.e. an increase in $n_{D^{-}}$is reflected in an increase in deuterium positive ion density. In the negative ion source of LHD-NBI, the extraction gap length and applied extraction voltage is not optimized for deuterium beam. Although the $n_{D^{-}}$increases, the $I_{a c c(D)}$ decreases because of the weak extraction field for deuterium negative ions. However, increasing the extraction field would probably cause an increase in the $I_{e}$, which is not desirable because of the consequent increase in the power to the extraction grid. Concern for this co-extracted electron is verified using a short extraction gap length in LHD-NBI in 2018, which will shown in section 5 .

Another confirmation is about the state of Cs on the PG surface which is the production surface of negative ions. Intensity from Cs neutral particle also increases 
for deuterium discharge. In order to verify the sputtering effect, we use a mixed gas discharge in the RNIS. The Cs neutral density in the vicinity of the PG is obtained by Cs laser absorption spectroscopy, as shown in Figure 3. Horizontal axis is the average mass number for mixture gas with helium with the percentages of $10 \%, 20 \%$, and $30 \%$ in hydrogen, which are plotted between hydrogen and the deuterium atomic mass numbers. The Cs neutral particle density is $2.2 \times 10^{14} \mathrm{~m}^{-3}$ in hydrogen discharge. That density increases to $6.5 \times 10^{14} \mathrm{~m}^{-3}$ for deuterium discharge. This increasing rate is consistent with the increasing of Cs optical emission signal measured by the OES. We also confirmed the increase of Cs density for hydrogen and He mixed plasma, which depended on the He fraction. Therefore, the Cs layer on the PG and the walls of the ion source is depleted by increased sputtering by deuterium compared to that with hydrogen because of the higher mass of deuterium. Maintaining the Cs layer on the PG in order to maintain the required negative ion production rate is one of the issues with operation in deuterium.

\section{Effect of Electron Suppression by Bias Voltage}

In the negative ion source of LHD-NBI, a magnetic filter is used for suppressing the inflow of electrons from the arc discharge region to the extraction region vicinity of the PG. However, the magnetic filter field is created by permanent magnets, and they are not easily changed. Therefore the bias voltage $\left(V_{\text {bias }}\right)$ applied between the ion source and the PG is used.

Figure 4(a) shows the change of the $I_{a c c(H)}$ and the $I_{e}$ for the $V_{\text {bias }}$ in the hydrogen operation in LHD-NBI. We normally use 2 - $3 \mathrm{~V}$ low bias voltage, which depends on the value of $I_{a c c(H)}$ and $I_{e} / I_{a c c(H)}$. The bias voltage suppresses not only electrons but also negative ions, see Figures 4 . Therefore, higher $V_{\text {bias }}$ is not an advantage for high power beam generation. In hydrogen discharge, a small increase in $I_{e}$ is observed even with a low $V_{\text {bias }}$. However, $I_{e} / I_{a c c(H)}$ is 0.3 when the $V_{\text {bias }}$ is $2 \mathrm{~V}$. Figure $4(\mathrm{~b})$ shows the dependence of the $n_{H^{-}}$, the $n_{e}$, and the plasma potential $(\phi)$ measured by the CRD, the mmIF, and the LP, respectively, on the $V_{\text {bias }}$ in the RNIS. In the case of the $3 \mathrm{~V}$ bias voltage, the $n_{H^{-}}$and the $n_{e}$ are $3 \times 10^{17} \mathrm{~m}^{-3}$ and $0.7 \times 10^{17} \mathrm{~m}^{-3}$, respectively. The density ratio of $n_{e} / n_{H^{-}}=0.23$ is consistent with $I_{e} / I_{a c c(H)}$ in LHD-NBI. The $n_{H^{-}}$and the $n_{e}$ each decrease as $V_{\text {bias }}$ increased. The $\phi$ increases from $1 \mathrm{~V}$ to $4 \mathrm{~V}$ by increasing the $V_{\text {bias }}(0-8 \mathrm{~V})$. Quasi neutrality in the plasma means that the decrease in the $n_{H^{-}}$ and the $n_{e}$ that accompany the increase in the positive potential of the PG must be accompanied by a decrease in the $\mathrm{H}^{+}$density. It is considered that the influx of electrons maintaining the charge neutrality also decreases in the extraction region at high bias voltage.

On the other hand, an increase in $n_{e}$ is clearly observed in the deuterium operation of the RNIS. Figure 5(a) shows the change of the $I_{a c c(D)}$ and the $I_{e}$ for the $V_{\text {bias }}$ in the deuterium discharge of the LHD-NBI. The $I_{a c c(D)}$ is saturated, and the electron current increases greatly at the $V_{\text {bias }}$ lower than $5 \mathrm{~V}$. The $I_{e} / I_{a c c(D)}$ is 0.7 with the low $V_{\text {bias }}$ of 
$2 \mathrm{~V}$, which is not a permissible value for safety beam operation to prevent damage to the EG surface due to electron current. Therefore, the $V_{\text {bias }}$ in the range of $5-6 \mathrm{~V}$ is the standard setting for deuterium beam operation in LHD-NBI. Figure 5(b) shows the dependence of the $n_{D^{-}}, n_{e}$, and $\phi$ on the $V_{\text {bias }}$ for deuterium discharge in the RNIS. The plasma potential of deuterium discharge is same as that in hydrogen discharge $(1-4 \mathrm{~V})$. The $n_{e}$ is larger than the $n_{D^{-}}$in the lower bias setting $\left(V_{\text {bias }}<2.5 \mathrm{~V}\right)$. These densities are the same value $\left(5 \times 10^{17} \mathrm{~m}^{-3}\right)$ at the $V_{\text {bias }}=2.5 \mathrm{~V}$, and the $n_{e}$ and the $n_{D^{-}}$are 5 times and 1.6 times higher than the $n_{e}$ and the $n_{H^{-}}$in hydrogen discharge, respectively. The $n_{e}$ decreases to $2.5 \times 10^{17} \mathrm{~m}^{-3}$ at the $V_{\text {bias }}=5.6 \mathrm{~V}$, which is sufficiently lower than the $n_{D^{-}}\left(4 \times 10^{17} \mathrm{~m}^{-3}\right)$ in the vicinity of the PG surface. If the $n_{H^{-}}$and $n_{D^{-}}$are the same in the vicinity of $\mathrm{PG}$ aperture, the maximum deuterium negative ion current should be limited to $1 / \sqrt{2}$ times the hydrogen negative ion current by Child-Langmuir law using the same extraction gap length with the same extraction voltage. Therefore, the $I_{a c c(D)}$ of $46 \mathrm{~A}$ in deuterium operation using $5.6 \mathrm{~V}$ bias voltage is consistent with the the product of the reduction rate (0.7) and the $I_{a c c(H)}$ of $70 \mathrm{~A}$ in hydrogen using the same arc discharge power (170 kW/source) in LHD-NBI. The density ratio of $n_{e} / n_{D^{-}}$ is 0.6 in RNIS, which is also comparable value with the $I_{e} / I_{a c c(D)}(0.2-0.6$ shown in section 5) in LHD NBI. Therefore, the ratio of $I_{e} / I_{a c c(D)}$ is closely related to the ratio of $n_{e} / n_{D^{-}}$in the vicinity of the PG. If the electron density in the vicinity of the PG can be maintained sufficiently lower than the negative ion density by the bias voltage, coextracted electrons can be reduced. Under this conditions, it is expected that a strong extraction electric field can increase the negative ion current while maintaining a low co-extracted electron. For that reason, we test a short gap length to extract deuterium negative ion beam in 2018 experimental campaign.

\section{Influence of Co-extracted Electron by Arc Discharge Power}

The thermal load of the beam accelerator caused by co-extracted electrons is an issue in high power and long pulse beam operation in N-NBI. The extracted electrons are bent by the electron deflection magnetic (EDM) field installed in the EG [5], and electrons are absorbed by the EG. Although the EG is cooled by a water flow, concentration of electrons causes damage to the EG surface in high power beam operation. For this reason, the influence of arc discharge power on negative ions and co-extracted electrons in the beam is investigated.

Figure 6 (a) shows the dependence of the $I_{a c c(H)}$ and $I_{a c c(D)}$ by two ion sources on the arc discharge power $\left(P_{\text {arc }}\right)$ in LHD-NBI. Since two negative ion beams are accelerated by one high voltage power supply in LHD-NBI, these current are the sum of two acceleration currents from the negative ion sources. The horizontal axis is the total $P_{\text {arc }}$ by two ion sources in Figures 6. Circle marks are the $I_{a c c(H)}$ and square marks are the $I_{a c c(D)}$ in 2017. The negative ion current linearly increases with respect to the $P_{a r c}$ and is not saturated. The averaged value of the ratio of $I_{a c c(D)} / I_{a c c(H)}$ is approximately 0.66 for the same arc discharge power. Star mark is the $I_{a c c(D)}$ using short extraction gap length 
(PG - EG : 7mm) in 2018. The bias voltage and filling pressure is set $5.6 \mathrm{~V}$ and $0.43 \mathrm{~Pa}$, respectively, these are the same as the settings in 2017. The $I_{a c c(D)}$ of $55.4 \mathrm{~A}$ with the averaged current density of $223 \mathrm{~A} / \mathrm{m}^{-3}$ is generated by two ion sources (Table 1 ). We can use high $P_{\text {arc }}$ of $400 \mathrm{~kW}$ (i.e. $200 \mathrm{~kW} /$ source) in the deuterium operation, which is same as the $P_{a r c}$ in hydrogen operation. The reason for increasing the $P_{\text {arc }}$ is reduction of the $I_{e} / I_{a c c(D)}$ in deuterium operation.

We also continue maintaining the low $I_{e}$ in deuterium as large as that in hydrogen beam using conservative bias and pressure setting, which contributes to safety beam operation in the first deuterium campaign in LHD. A strong dependence of $I_{e} / I_{a c c(D)}$ on the $P_{\text {arc }}$ appeared in the deuterium beam operation, as shown in Figure 6(b). In the case of hydrogen operation, the $I_{e} / I_{a c c(H)}$ distribute between 0.2 and 0.3 . The lower limit of $I_{e} / I_{a c c(H)}$ slightly increases as increasing $P_{\text {arc }}$, which corresponds to the optimum operation in which electrons are suppressed with the maximum beam current. In deuterium discharge, $I_{e} / I_{a c c(D)}$ are widely distributed $(0.2-0.6)$ which including the adjusted phase data using lower $P_{\text {arc }}(100$ - $150 \mathrm{~kW} /$ source $)$ while investigating the dependency of $V_{b i a s}$ and filling pressure. The lower limits of $I_{e} / I_{a c c(D)}$ are 0.2 in both hydrogen and deuterium discharges in $100 \mathrm{~kW} /$ source. However, the $I_{e} / I_{a c c}(D)$ increases greatly with the increase of the arc power, which is the unique characteristic in deuterium operation. The $I_{e} / I_{a c c(D)}$ increases to 0.39 at the $P_{a r c}=170 \mathrm{~kW} /$ source in high $I_{a c c(D)}$ operation, which is twice larger than the $I_{e} / I_{a c c(H)}$ in hydrogen operation using same $P_{\text {arc }}$. The thermal load on the EG due to co-extracted electron is in a safety level in the operations near the lower limit of $I_{e} / I_{a c c(D)}$ in this operation. However, the gradient of the slope is 6.4 times higher than that of hydrogen, thus if the ion source will be operated using higher arc discharge power, the thermal load due to electrons will become a problem. Thus, this trend is an important issue for considering the production of high-power deuterium beam in every N-NBI. Star mark is the result of $I_{e} / I_{a c c(D)}$ using short gap length $(7 \mathrm{~mm})$ in 2018. The $I_{e} / I_{a c c(D)}$ decreases to 0.31 in the $P_{\text {arc }}$ of 200 $\mathrm{kW} /$ source with the $5.6 \mathrm{~V}$ bias voltage and the $0.43 \mathrm{~Pa}$ filling pressure. The $I_{e} / I_{a c c(D)}$ using short gap length is much lower than the lower limit value of $I_{e} / I_{a c c(D)}$ in 2017 , which is almost equal to the $I_{e} / I_{a c c(H)}$ in hydrogen operation. Since the $I_{e} / I_{a c c(D)}$ ratio can be maintain low, high arc power operation for increasing the $I_{a c c(D)}$ current become possible.

Figure 7 shows changes in negative ion density and electron density for arc discharge power in the RNIS. The negative ion densities increase as the arc power increases, and the $n_{D^{-}}$is higher than the $n_{H^{-}}$. It is considered that the ionization and the dissociation in the discharge region increase, and neutral particles and positive ions which are the sources of negative ions, increase. Since the mass of deuterium is heavier than hydrogen, the diffusion coefficient is lower and the confinement of deuterium in the vicinity of the PG should be improved. On the other hand, the electron density also increases as the arc power increases, but that density differs greatly between hydrogen discharge and deuterium discharge. The electron density in deuterium discharge is 4 times larger than the electron density in hydrogen discharge. The density fraction of $n_{e} / n_{H^{-}}$is as low 
as 0.17 in $60 \mathrm{~kW}$ discharge power, but the $n_{e} / n_{D^{-}}$is 0.61 . Although the result of the $n_{e} / n_{D^{-}}$is slightly larger than the $I_{e} / I_{a c c(D)}$ ratio range shown in Figure $6(\mathrm{~b})$, there is the common tendency of electron increasing. Therefore, an increase in electron density due to a high arc power is considered to be responsible for an increase in the $I_{e} / I_{a c c(D)}$ in deuterium discharge.

Here, we must discuss a distribution of negative ion density and electron density perpendicular to the PG surface to understand electron contamination in the beam. We obtained flow direction of negative hydrogen ion during beam extraction by laser assisted directional Langmuir probe in RNIS [26,30]. According to the result, the turning point of negative hydrogen ion which released from the PG surface return to the PG direction was approximately $20 \mathrm{~mm}$ from the PG. This was also confirmed by the twodimensional distribution of the extraction of negative hydrogen ion measured by the $\mathrm{H}_{\alpha}$ imaging diagnostic $[31,32]$. The extraction distribution of hydrogen negative ions was strongly distributed near the $\mathrm{PG}$ aperture in the range of $0<z<20 \mathrm{~mm}$, here $\mathrm{z}$ is the distance from the PG surface, which is closely related to the distribution of EDM field in the vicinity of PG surface. A negative ion rich plasma with low electron is formed in the region between $\mathrm{PG}$ and EDM lobe $[23,30]$. The negative ion density should decrease outside the turning point of negative ions, and it is considered that the inflow of electrons from the discharge region increases. In the case of deuterium discharge, the velocity of the $\mathrm{D}^{-}$ions generated from the $\mathrm{PG}$ should be 0.7 times of $\mathrm{H}^{-}$velocity if both ions have the same energy. If the turning point of the deuterium negative ion is closer to the PG, then the area with high electron density should be closer to the PG. The reduction in the electron density and control of its distribution in the vicinity of the PG is one of the key parameters to reduce the co-extracted electrons current.

On the other hand, the response of the $n_{H^{-}}$, the $n_{D^{-}}$and the $n_{e}$ to the $V_{\text {bias }}$ to suppress co-extracted electrons is very interesting. It is well known that electron current extracted from a volume-production negative ion source increases with deuterium discharge [33]. Since the decay curve of the electron current for bias voltage was shifted by about $2 \mathrm{~V}$, it was presumed that the change was due to the difference in the plasma potential. However, in the surface-production negative ion source of the RNIS, there is no difference in plasma potential between hydrogen and deuterium discharge. As the bias voltage decreases, the plasma potential decreases. However the electron density and the negative ion density increase in lower plasma potential. Since the sum of the electron density and the negative ion density is strongly related to the positive ion density by the charge neutrality, the $\mathrm{D}^{+}$ion density in the deuterium discharge is larger than the $\mathrm{H}^{+}$ion density in the same bias condition. Increase of $\mathrm{D}^{+}$ions is because the decrease of $\mathrm{D}^{+}$ion velocity owing to weight of the $\mathrm{D}^{+}$is twice as heavy. In the surface-production negative ion source, most of the negative ions in the vicinity of the PG are supplied from the PG surface, so that electrons accompanying the positive ions from the discharge region bridge the gap between the negative ion density and the positive ion density by charge neutrality. By suppressing positive ion density at high bias voltage, electrons entering along with positive ions are also suppressed. Therefore, it is considered that control of 
positive ions in the vicinity of the PG is also important factor to suppress co-extracted electrons.

\section{Conclusion}

Deuterium negative ion beam operation for LHD-NBIs was initiated in 2017. The $I_{a c c(D)}$ of $46 \mathrm{~A}$ has been extracted for $2 \mathrm{sec}$ with the averaged current density of 190 $\mathrm{A} / \mathrm{m}^{2}$ by two negative ion sources in the injector in first deuterium campaign. The ratio of $I_{e} / I_{a c c(D)}$ was 0.39 . Although the configuration of the ion source was not optimized for deuterium, the observed $I_{a c c(D)}$ reached $82 \%$ of the LHD requirement in the first phase deuterium beam operation, and those results were comparable to the ITER-NBI specification (40 A negative ion current with the current density of $200 \mathrm{~A} / \mathrm{m}^{2}$ at $0.3 \mathrm{~Pa}$ ). Linear dependence of the minimum value of the $I_{e} / I_{\text {acc }(D)}$ on the $P_{\text {arc }}$ was found, and was stronger than the $I_{e} / I_{a c c(H)}$, which is one of the issue for deuterium operation. A clear increase in electron density in the vicinity of the PG was confirmed by the RNIS. Changes in electron density and negative ion density at the switching of operation gas from hydrogen to deuterium in the RNIS agree with the trend of beam current in the LHD-NBI. A bias voltage satisfying the condition of $n_{D^{-}}>n_{e}$ in the vicinity of the PG realizes a high $I_{a c c(D)}$ and a low $I_{e}$ using a strong extraction field between the PG and the EG. The $I_{a c c(D)}$ current of $55.4 \mathrm{~A}$ (averaged current density : $233 \mathrm{~A} / \mathrm{m}^{2}$ is achieved

for $1.5 \mathrm{sec}$ using the short gap length $(7 \mathrm{~mm})$ in 2018 . The lower $I_{e} / I_{a c c(D)}$ of 0.31 is maintained by $200 \mathrm{~kW} /$ source high-discharge power operation, which becomes the same level as the $I_{e} / I_{a c c(H)}$ in hydrogen operation.

\section{Acknowledgments}

The authors would like to thank the technical staffs of LHD and NBI for their great contributions. This research is supported by the budget for the NIFS No. ULRR702.

\section{References}

[1] M. Kuriyama et al , Fusion Tech. 1996 (Proc. 19th Symp. Fusion Tech. Lisbon, 16-20 Sep. 1996) (1996) 693 - 696 .

[2] A. Kojima et al, Nucl. Fusion 51, (2011) 083049.

[3] A. Iiyoshi et al, Nucl. Fusion 39, (1999) 1245.

[4] O. Kaneko et al, Nucl. Fusion 43, (2003) 692.

[5] Y. Takeiri et al, Rev. Sci. Instrum. 71, (2000) 1225.

[6] K. Tsumori et al, Rev. Sci. Instrum. 75, (2004) 1847.

[7] T. Mutoh et al, Fusion Science and Technology 68, (2015) 216 - 224.

[8] K Tsumori et al, Plasma Sci. Technol. 8 (2006) 24.

[9] R. Hemsworth, A. Tanga and V. Antoni, Rev. Sci. Instrum. 79, (2008) 02C109.

[10] M. J. Singh et al, New J. Phys. 19, (2017) 055004.

[11] P. Sonato et al, Fusion Eng. Des. 84, (2009) 269 - 274.

[12] V. Toigo et al, New J. Phys. 19, (2017) 085004.

[13] W. Kraus et al, Rev. Sci. Instrum. 89, (2018) 052102. 
[14] K. Ikeda et al , AIP Conference Proceedings 2011, (2018) 060002.

[15] Y. Takeiri et al, Rev. Sci. Instrum. 68, (1997) 2003.

[16] Y. Takeiri et al, Fusion Sci. Technology 58, (2010) 482 - 488.

[17] M. Osakabe et al , IEEE Transactions on Plasma Science 46, (2018) 2324 - 2331.

[18] M. Osakabe, et al, Fusion Sci. Technology, 72, (2017)199 - 210.

[19] M. Kobayashi, et al , Fusion Eng. Des., 137, (2018) 191-195.

[20] Y. Takeiri et al, Nucl. Fusion 46, (2006) S199 - S210.

[21] M. Kisaki et al , Rev. Sci. Instrum. 87, (2016) 02B321.

[22] K. Tsumori et al, Plasma Sci. Technol. 8 (2006) 24.

[23] K. Tsumori and M. Wada, New J. Phys. 19, (2017) 045002.

[24] H. Nakano et al, AIP Conference Proceedings 1390, (2011) 359.

[25] T. Tokuzawa et al, Rev. Sci. Instrum. 87, (2016) 11E105.

[26] S. Geng et al, Rev. Sci. Instrum. 87, (2016) $02 \mathrm{~B} 103$.

[27] K. Tsumori et al , AIP Conference Proceedings 1869, (2017) 030001.

[28] K. Ikeda et al , AIP Conference Proceedings 1390, (2011) 367.

[29] K. Ikeda et al , AIP Conference Proceedings 1869, (2017) 050004.

[30] S. Geng et al , Fusion Eng. Des. 123, (2017) 481 - 484.

[31] K. Ikeda et al, New J. Phys. 15, (2013) 103026.

[32] K. Ikeda et al , AIP Conference Proceedings 1655, (2015) 040005.

[33] T. Inoue et al, Rev. Sci. Instrum. 61, (1990) 496-498. 
Table 1. Setting of extraction gap length and the results of the negative ion current and the electron-ion current ratio in in LHD-NBI

\begin{tabular}{llllllll}
\hline Year & Beam line & Ext. gap & GG type & $I_{a c c(H)}$ & $I_{e} / I_{a c c(H)}$ & $I_{a c c(D)}$ & $I_{e} / I_{a c c(D)}$ \\
\hline 2017 & BL1 & $7 \mathrm{~mm}$ & Slot & $81.5 \mathrm{~A}$ & 0.27 & $41.6 \mathrm{~A}$ & 0.49 \\
2017 & BL2 & $8 \mathrm{~mm}$ & Aperture & $74.2 \mathrm{~A}$ & 0.30 & $45.5 \mathrm{~A}$ & 0.54 \\
2017 & BL3 & $8 \mathrm{~mm}$ & Slot & $77.5 \mathrm{~A}$ & 0.23 & $46.2 \mathrm{~A}$ & 0.39 \\
2018 & BL3 & $7 \mathrm{~mm}$ & Slot & $73.9 \mathrm{~A}$ & 0.25 & $55.4 \mathrm{~A}$ & 0.31 \\
\hline
\end{tabular}




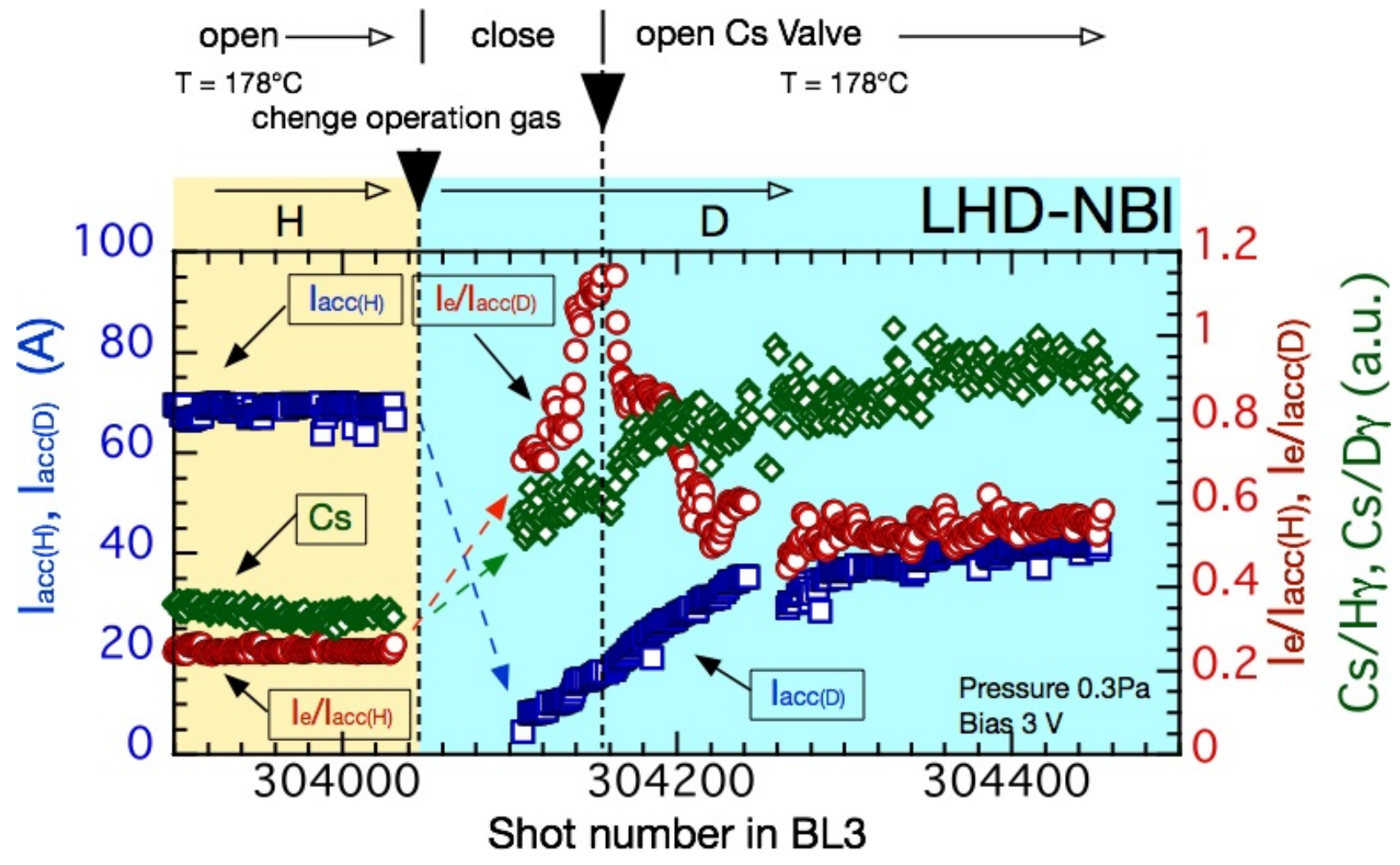

Figure 1. Shot history of the $I_{a c c(H)}$ and $I_{a c c(D)}$ (squares), $I_{e} / I_{a c c(H)}$ and $I_{e} / I_{a c c(D)}$ (circles), and the optical signal of Cs neutral (diamonds) during the operation gas changing from $\mathrm{H}_{2}$ to $\mathrm{D}_{2}$ in the negative ion source. The $P_{\text {arc }}$ is $160 \mathrm{~kW} /$ source in hydrogen discharge. Deuterium beam operation initiated $P_{\text {arc }}=50 \mathrm{~kW}$ and increased to $120 \mathrm{~kW} /$ source. Deuterium negative ion current decreased to $60 \%$ of hydrogen ion current in the first trial. The $I_{e} / I_{a c c(D)}$ largely increased by deuterium discharge, but it recovered by additional Cs feeding. Reproduced from Figure 2 in AIP Conference Proceedings 2011, 060002 (2018); https://doi.org/10.1063/1.5053331, with the permission of AIP Publishing. 
Exploring Deuterium Beam Operation in Negative-Ion-Based Neutral Beam Injector 15

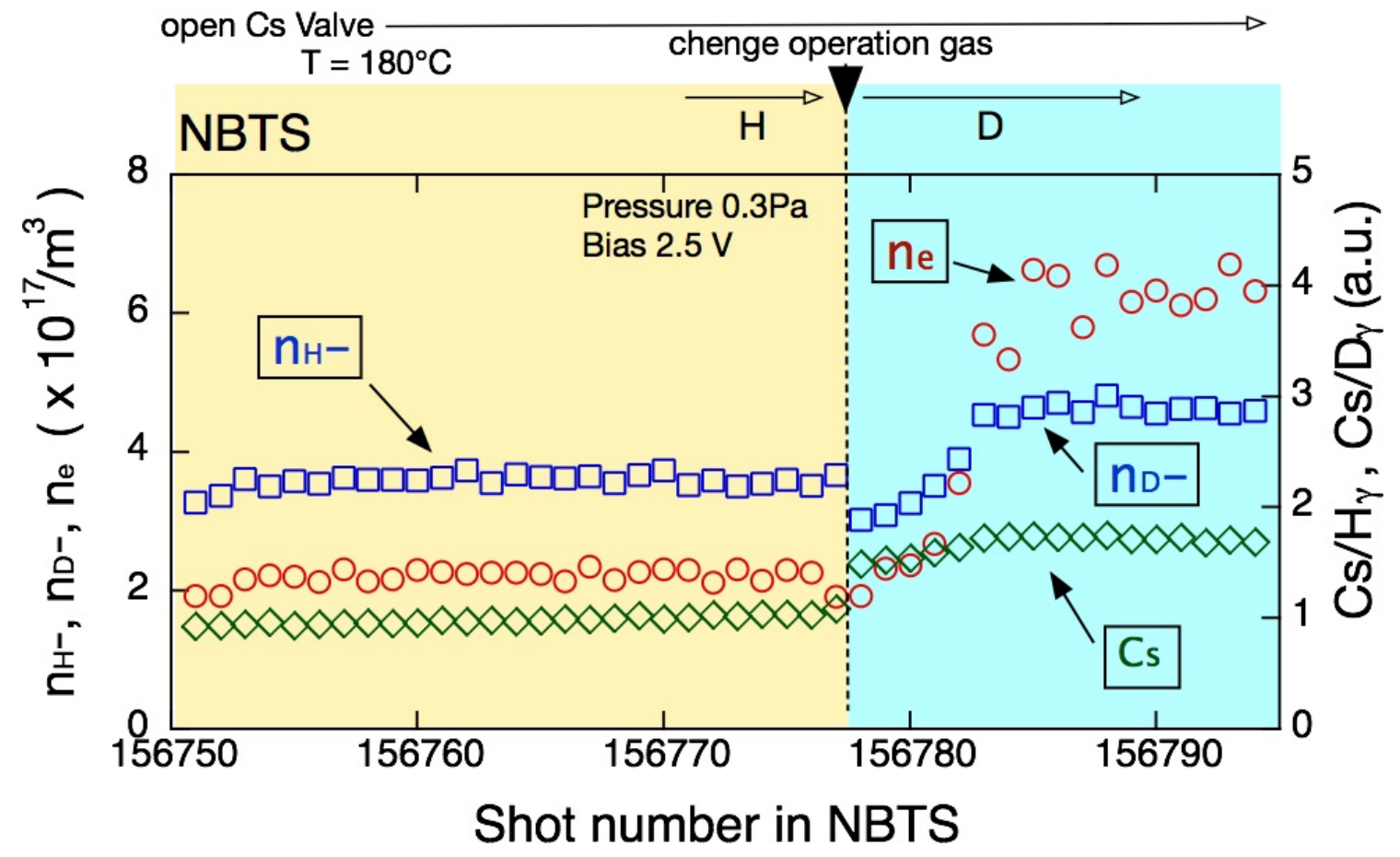

Figure 2. Shot history of the $n_{H^{-}}$and $n_{D^{-}}$(squares), $n_{e}$ (circles), and the optical signal of Cs neutral (diamonds) in the vicinity of PG surface during the operation gas changing from $\mathrm{H}_{2}$ to $\mathrm{D}_{2}$ in the RNIS. The $P_{\text {arc }}$ is $50 \mathrm{~kW}$ in hydrogen and deuterium discharge, which is temporarily reduced to $30 \mathrm{~kW}$ in a few shots immediately after gas change. 
Exploring Deuterium Beam Operation in Negative-Ion-Based Neutral Beam Injector 16

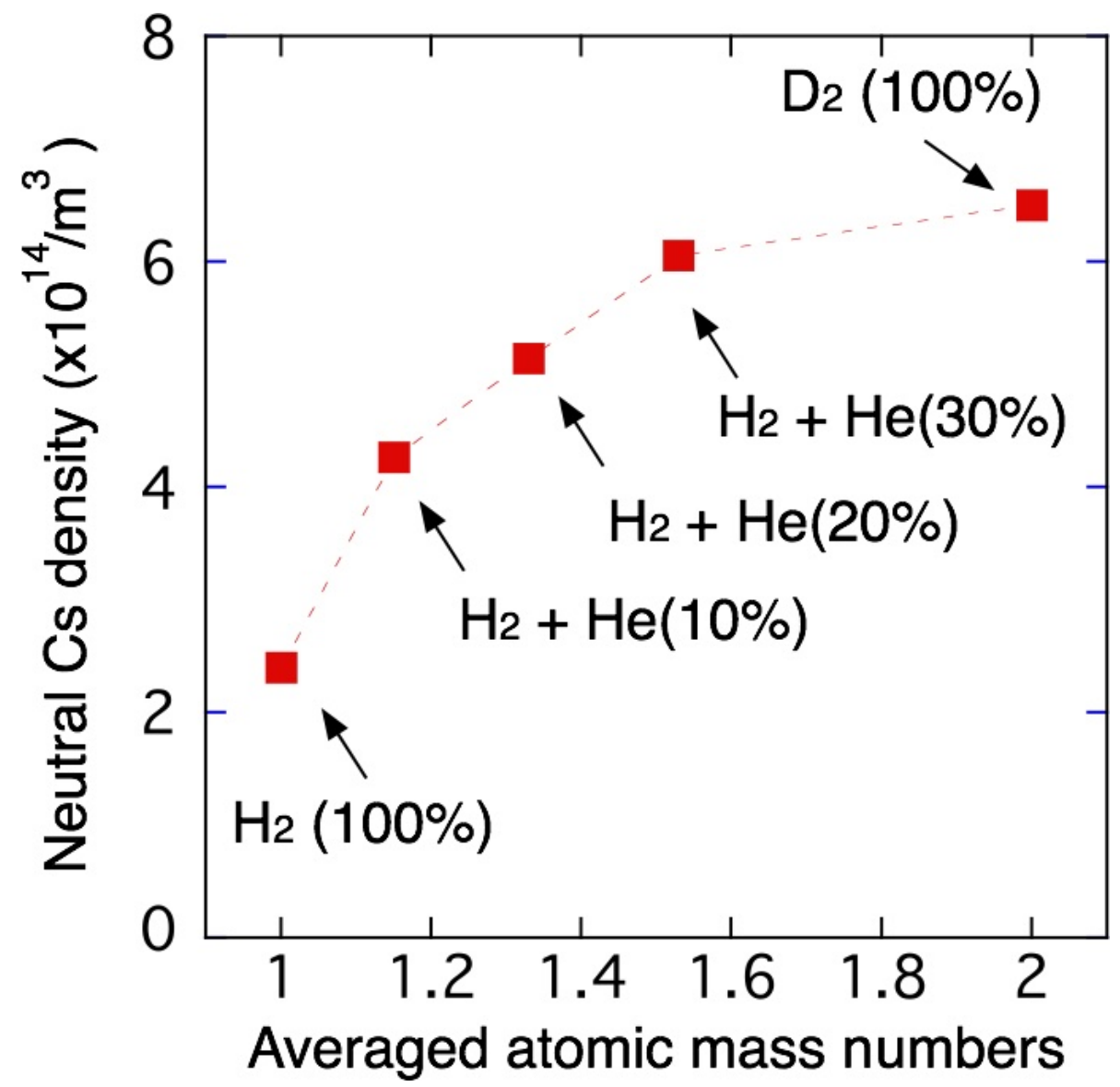

Figure 3. Neutral Cs density measured by the Cs laser absorption diagnostic for hydrogen, deuterium, and helium mixed hydrogen discharge with low discharge power of $P_{\text {arc }}=28 \mathrm{~kW}$ in the RNIS. 
(a) NBI in LHD

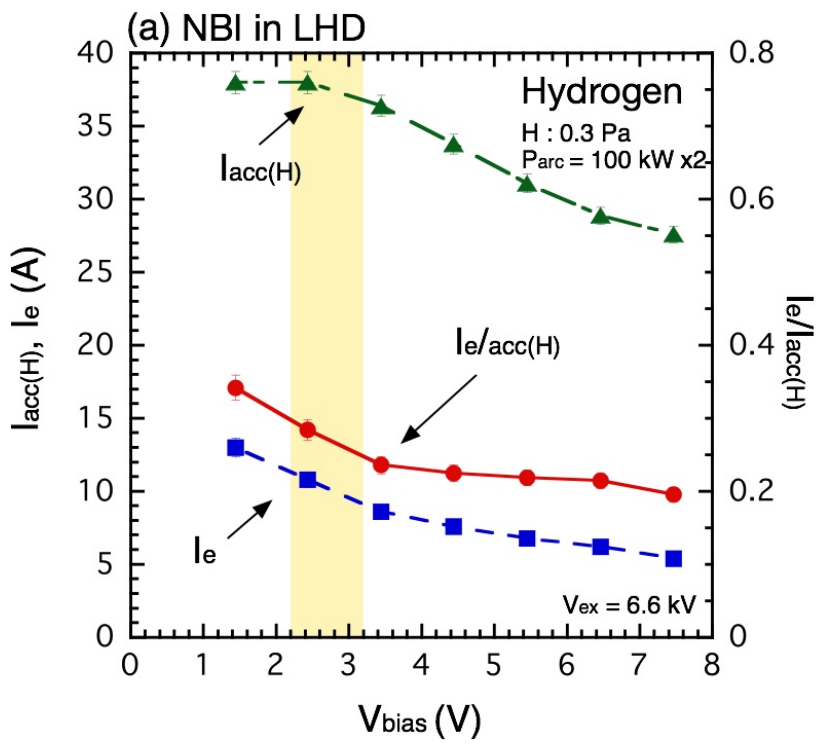

(b) RNIS in NBTS

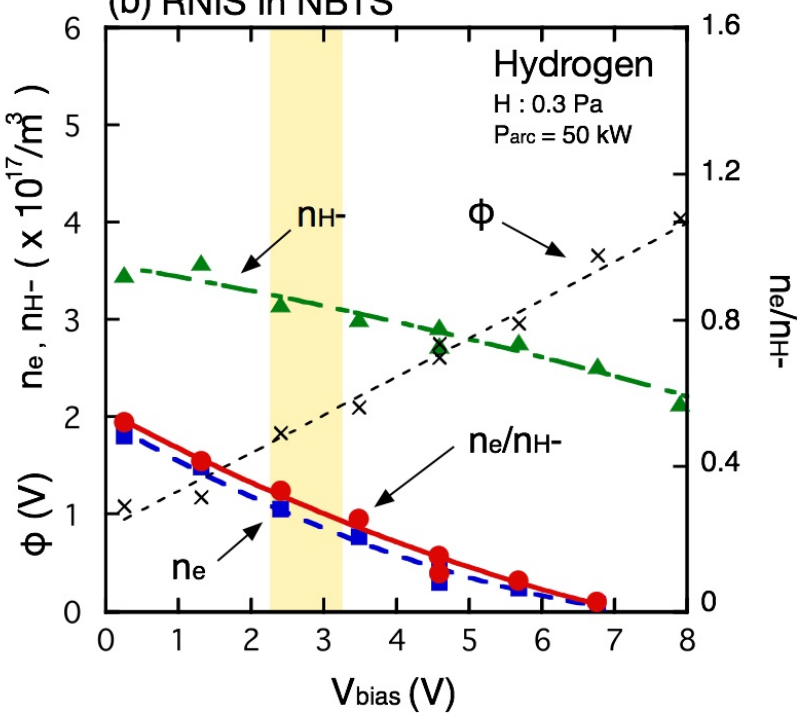

Figure 4. (a) Dependence of the $I_{a c c(H)}$ (triangle), the $I_{e}$ (square), and the $I_{e} / I_{a c c(H)}$ (circle) in LHD-NBI on the bias voltage. Reproduced from Figure 3 in AIP Conference Proceedings 2011, 060002 (2018); https://doi.org/10.1063/1.5053331, with the permission of AIP Publishing. (b) Dependence of the $n_{H^{-}}$(triangle), the $n_{e}$ (square), the $n_{e} / n_{H^{-}}$(circle), and the plasma potential $\phi$ (cross) at $19 \mathrm{~mm}$ from the PG in the RNIS in the NBTS on the bias voltage. Shaded area is the range of the $V_{\text {bias }}$ setting that is normally used for the hydrogen operation in LHD-NBI. 
Exploring Deuterium Beam Operation in Negative-Ion-Based Neutral Beam Injector 18

(a) NBI in LHD

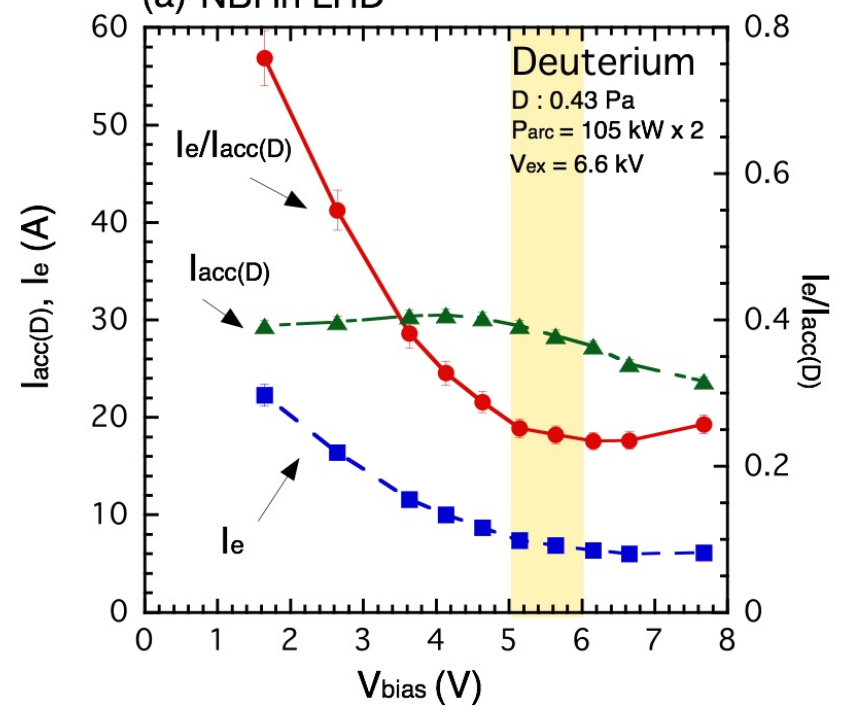

(b) RNIS in NBTS

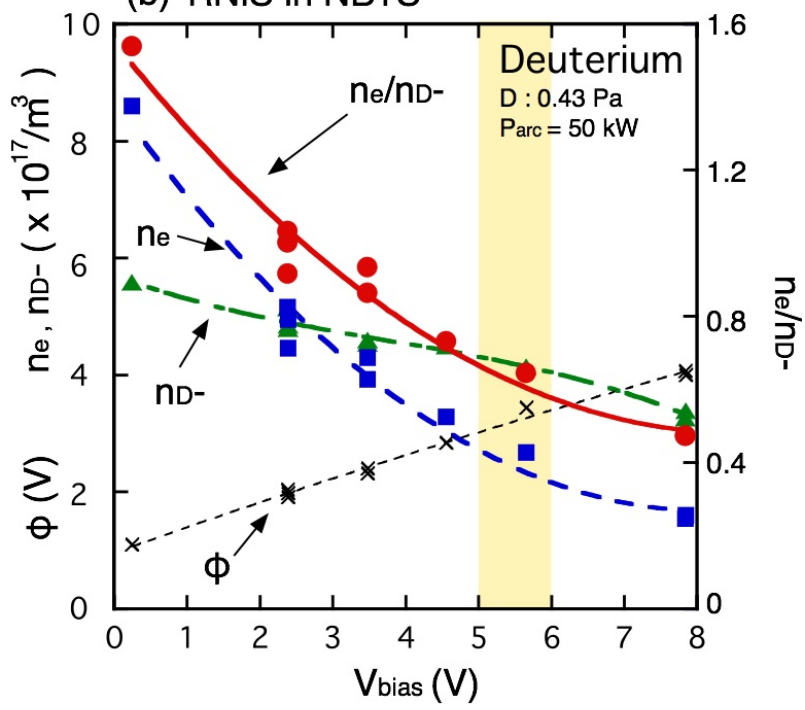

Figure 5. (a) Dependence of the $I_{a c c(D)}$ (triangle), the $I_{e}$ (square), and the $I_{e} / I_{a c c(D)}$ (circle) in LHD-NBI on the bias voltage. Reproduced from Figure 3 in AIP Conference Proceedings 2011, 060002 (2018); https://doi.org/10.1063/1.5053331, with the permission of AIP Publishing. (b) Dependence of the $n_{D^{-}}$(triangle), the $n_{e}$ (square), the $n_{e} / n_{D^{-}}$(circle), and the $\phi$ (cross) at $19 \mathrm{~mm}$ from the PG in the RNIS in the NBTS on the bias voltage. Shaded area is the range of the $V_{\text {bias }}$ setting that is normally used for the deuterium operation in LHD-NBI. 


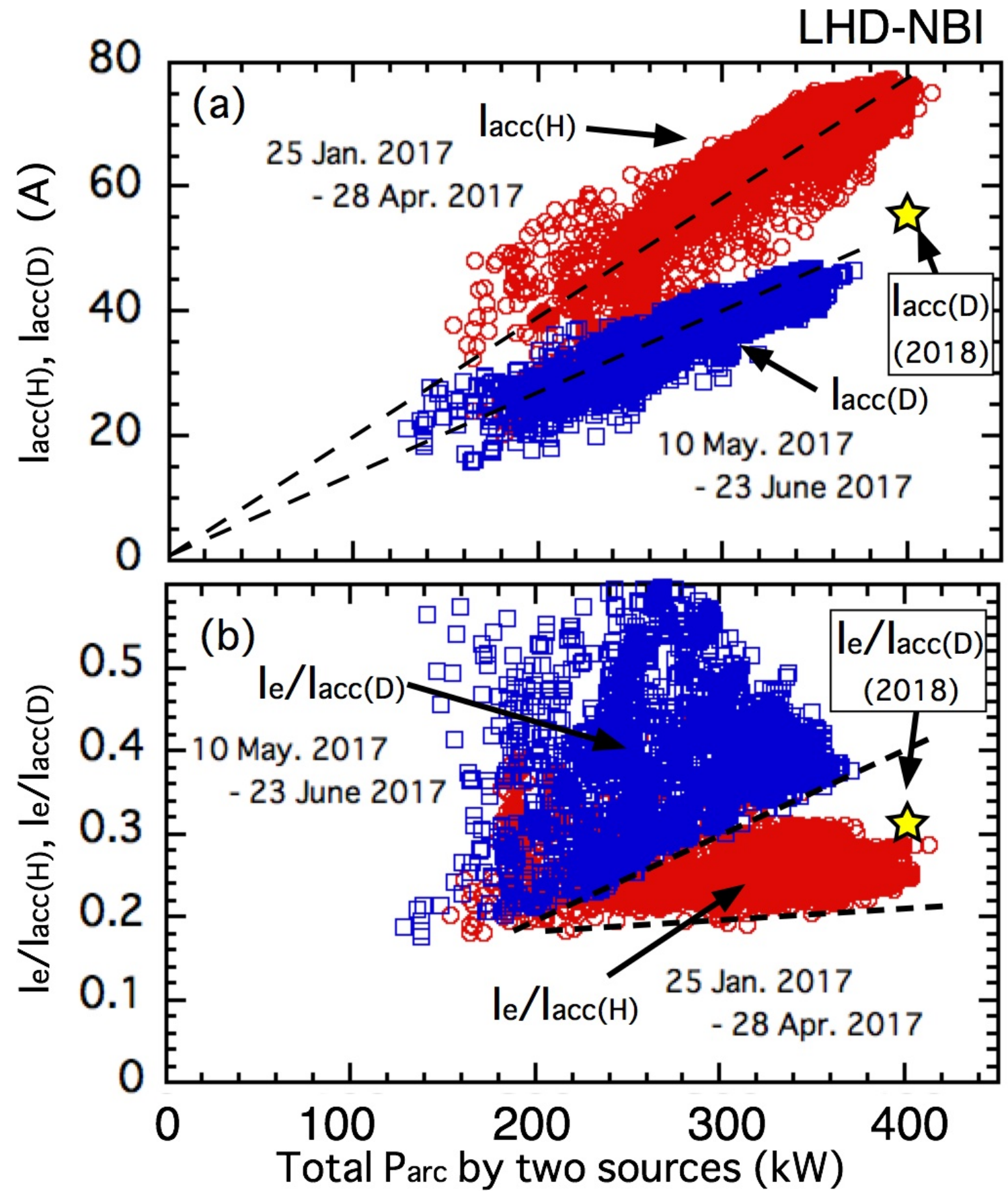

Figure 6. Dependence of (a) the $I_{a c c(H)}$ and the $I_{a c c(D)}$, (b) the $I_{e} / I_{a c c(H)}$ and the $I_{e} / I_{a c c(D)}$ on the total $P_{a r c}$ by 2 ion sources for hydrogen discharge (circle) and deuterium discharge(square) using $8 \mathrm{~mm}$ extraction gap length optimized for $\mathrm{H}^{-}$ operation in LHD-NBI in 2017. Star marks are the results of $\mathrm{D}^{-}$operation using short gap length $(7 \mathrm{~mm})$ in 2018. Reproduced from Figure 4 in AIP Conference Proceedings 2011, 060002 (2018); https://doi.org/10.1063/1.5053331, with the permission of AIP Publishing. 


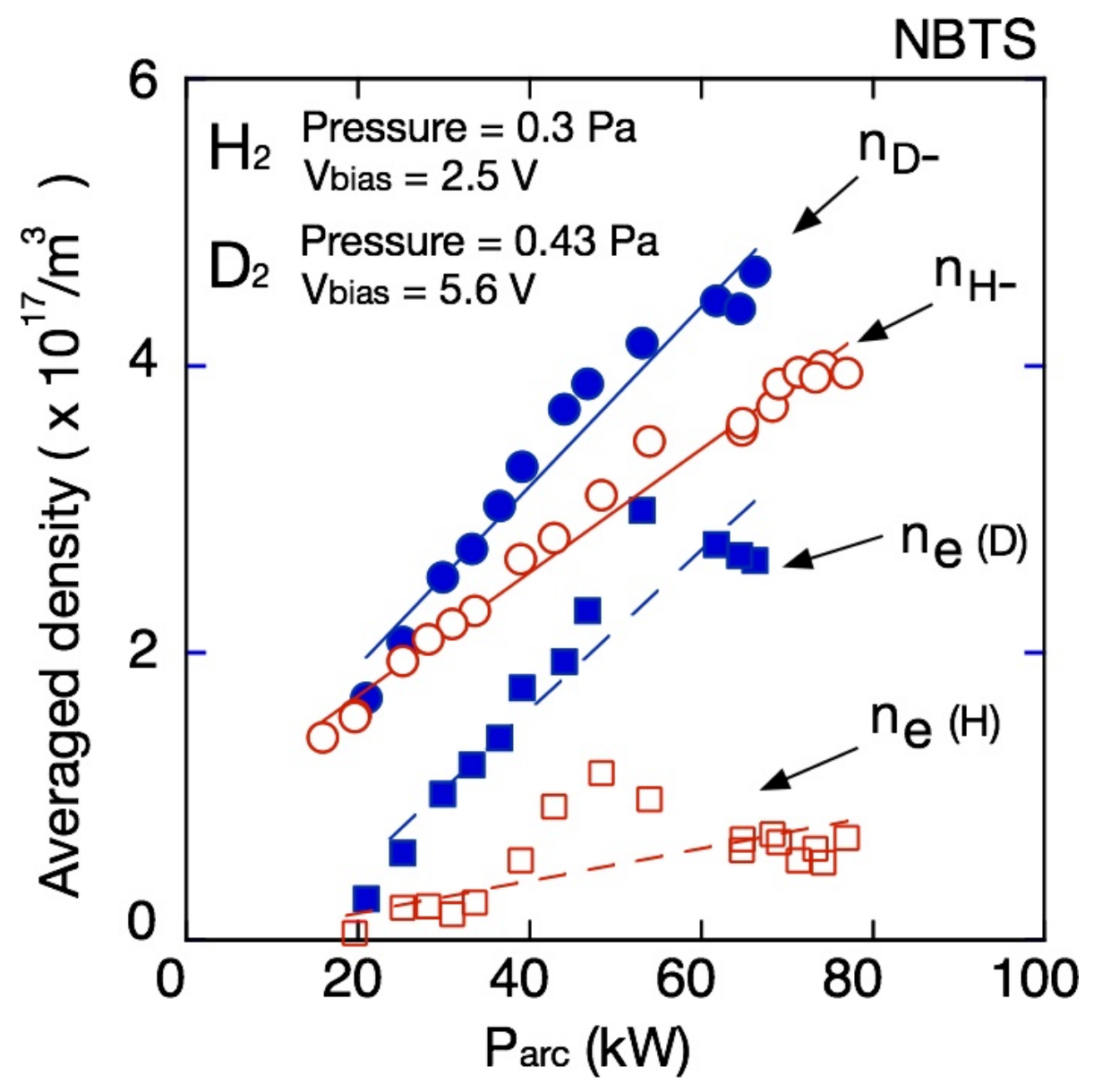

Figure 7. Dependence of the negative ion density (circle) and electron density (square) for hydrogen discharge (open) and deuterium discharge (closed) in the RNIS in the NBTS on the arc discharge power. 